\title{
Feasibility Study of HHT Method on Helicopter Rotor Imbalance Fault Feature Extraction
}

\author{
Wen $\mathrm{Cao}^{1}$, Zhen-hong Xing ${ }^{2}$, Yi Yao ${ }^{3}$, Meng Zhang ${ }^{4}$ \\ ${ }^{1,3,4}$ Vehicle Engineering Department, Changchun Institute of Engineering and Technology, china \\ ${ }^{2}$ Changping NCO School Academy of Equipment, china
}

Keywords: HHT, EMD, Helicopter Rotor imbalance fault, Feature extraction.

\begin{abstract}
Firstly, using the Hilbert-Huang Transform (HTT) methods of data for the empirical mode decomposition (EMD), so as to extract the Intrinsic mode function (IMF) of the signal, and then do Hilbert transform to get the Hilbert spectrum of the signal. The method has great advantages to non-linear and non-stationary data signal. For helicopter rotor imbalance fault causes the body to the vibration characteristics of the data, the marginal spectrum analysis its, can get the valid fault feature.
\end{abstract}

\section{Introduction}

In the current helicopter fault diagnosis system, most applications data analysis method based on feature extraction. Failure of the rotor unbalance, vibration signals can be extracted space on the body and deliver it to the analysis of its characteristics to achieve fault diagnosis ${ }^{[1]}$. In the current application, for the analysis of vibration signals mainly methods FFT、 wavelet analysis. Generation process failure and collected signal noise and other signals such that the actual collected helicopter airframe vibration signal exhibits a non-linear, non-stable, non-stable.The traditional method has difficulty for non-stationary signals, so that these methods are inadequate for the presence of non-steady-signal applications, As FFT analysis requires data on stationary, wavelet analysis it is actually adjustable window Fourier transform, And difficulties since the adaptability of wavelet selection $^{[3]}$.

When the Hilbert transform method of EMD (HHT) is based on a self-adaptive frequency analysis method. It can be based on the local characteristics of the signal, adaptive modal decomposition, overcomes the shortcomings of traditional methods, and in the process to achieve a non-stationary signals filtering ${ }^{[2]}$.

\section{HHT Method Principle}

\section{EMD decomposition.}

According to the meaning of the instantaneous frequency, not all the instantaneous frequency of the signal can be discussed, Only when the signal has the formula:

$$
X(t)=A(t) e^{j \varphi(t)}
$$

In order to calculate the instantaneous frequency of the form.

Based on the assumption: any complex signals are different from the composition of intrinsic mode functions, were intrinsic mode decomposition, each intrinsic mode function qualifying:

(1) The entire data in the number of extreme points is equal to the number of zero crossings and no more than one or at most;

(2) At any time, the envelope formed zero mean local maxima;

Therefore, the signal EMD decomposition of the instantaneous signal is present in only one of each frequency component of the multi-component form groups IMF.According to the EMD process, where $C_{k}$ is the intrinsic mode components, $r_{n}$ is a monotonic function, called residual function.

Finally get: 


$$
X(t)=\sum_{k=1}^{n} C_{k}+r_{n}
$$

According to the IMF found that the physical meaning, its every moment there is only one frequency, which can calculate the instantaneous frequency of its, this process also has the physical meaning of the instantaneous frequency.

\section{Hilbert spectrum and Hilbert marginal spectrum.}

EMD local characteristic time scale based on the signal, adaptive signal decomposition of $n-1$ component and the remaining functions of the IMF and , then calculate the instantaneous frequency and amplitude of each IMF component.

Hilbert transform for each mode functions:

$$
\hat{C}(t)=\frac{1}{\pi} P \int_{-\infty}^{+\infty} \frac{C(\tau)}{t-\tau} d_{\tau}
$$

Structural analysis signal:

$$
Z(t)=C(t)+j \hat{C}(t)=A(t) e^{j \varphi(t)}
$$

Thus available:

$$
X(t)=\operatorname{Re} \sum_{i=1}^{n} A_{i}(t) e^{j \varphi_{i}(t)}=\operatorname{Re} \sum_{i=1}^{n} A_{i}(t) e^{j \int \omega_{i}(t) d t}
$$

Where Re is taking the real part, equation omitted residual items $r_{n}$.

$$
H(\omega, t)=\operatorname{Re} \sum_{i=1}^{n} A_{i}(t) e^{j \int \omega_{i}(t) d t}
$$

Hilbert marginal spectrum is defined as:

$$
h(\omega)=\int_{0}^{T} H(\omega, t) d t
$$

$\mathrm{T}$ is the signal length. $\mathrm{H}(\omega, \mathrm{t})$ describes the change in the amplitude of the signal over the entire frequency range over time. $\mathrm{H}(\omega)$ reflected the changes in amplitude of the signal over the entire frequency range with frequency. Marginal spectrum represents the accumulated data in this frequency, unlike the steady-state distribution of the Fourier frequency.

\section{Simulation Signal Analysis}

A Using HHT Method, of formula (8) as shown in the simulation.

$$
Y=\sin (30 \pi t)+10 t \cos [70 \pi t+\sin (20 \pi t)]+\sin \left(10 \pi t^{2}\right)
$$

Analysis shows, its frequency components into three parts: $15 \mathrm{HZ}$ sinusoidal signal、35Hz amplitude increases with time as the center of the FM signal and the frequency increases with time varying component. Fig. 1 shows the Fourier spectrum of the peak frequency $15 \mathrm{HZ}, 25 \mathrm{~Hz}, 35 \mathrm{~Hz}$ and $45 \mathrm{~Hz}$ in, and an excess of $60 \mathrm{~Hz}$ frequency components. This is because the nonlinear signal, Such as FFT, Wavelet like approach requires higher-order harmonics to simulate nonlinear process, resulting in a false frequency components .
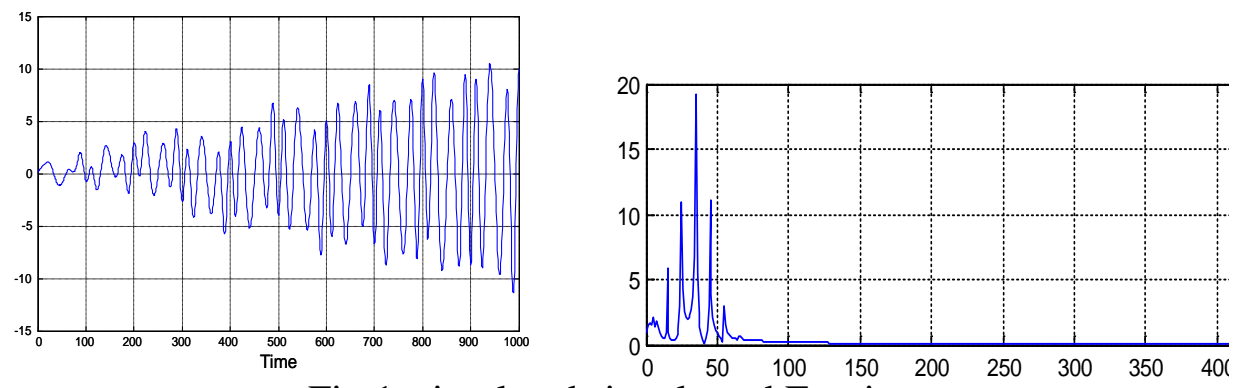

Fig.1. simulated signals and Fourier

By EMD decomposed into intrinsic mode components (Fig.2), top-down with time in order to reduce the frequency components, the remaining components which function as a monotonic 
function of frequency analysis does not affect.
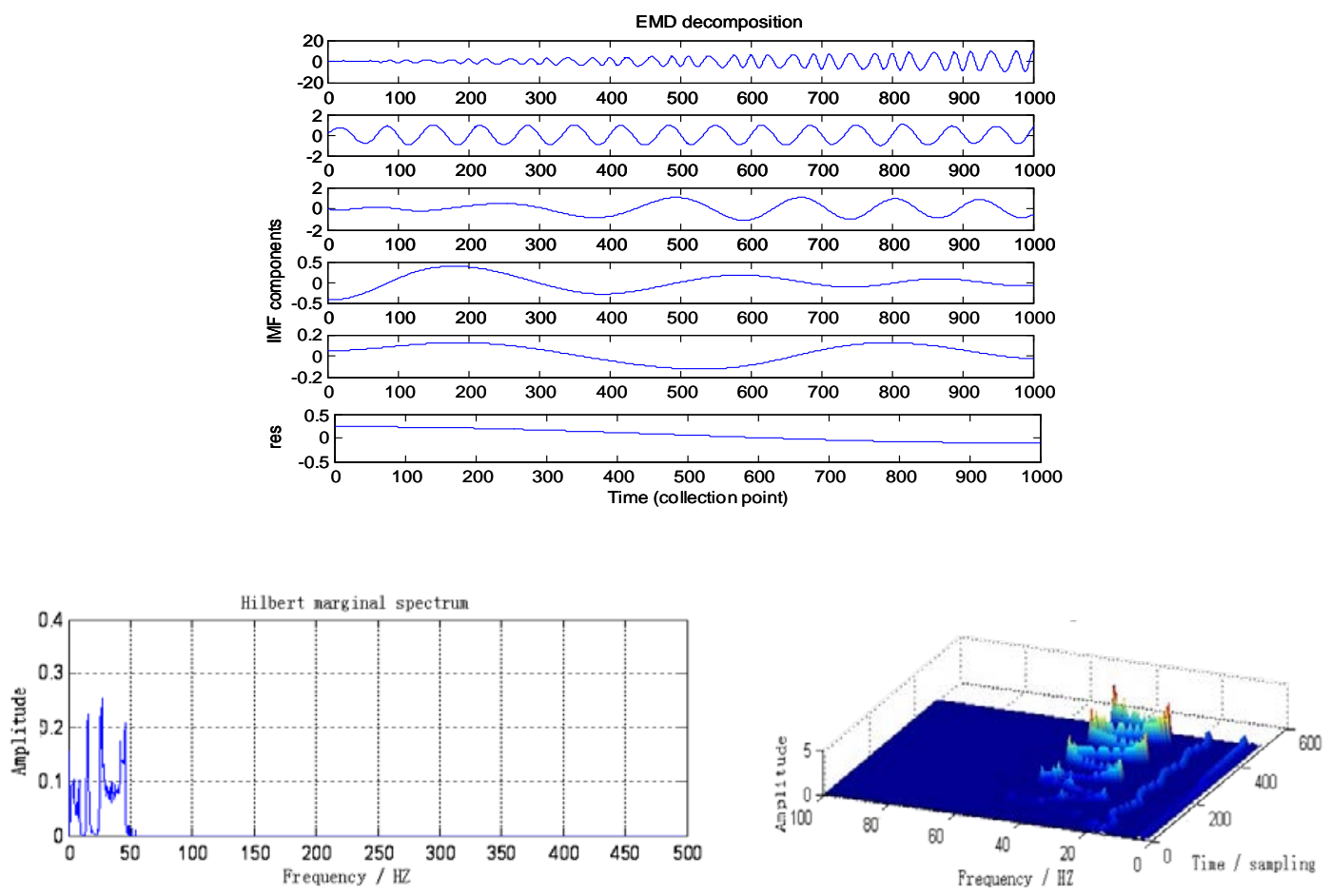

Fig. 2 Emulation signal EMD decomposition, Hilbert marginal spectrum and spectrum

By Hilbert and Hilbert spectrum when the spectrum can be obtained, and the amplitude of the oscillation signal changes over time. Process at sampling time (sample point) changes from 0 to 500 in, you can also clearly see the frequency increases over time, the frequency of the signal components.

Can be obtained from the simulation analysis, Fourier transform in the analysis of non-steady signal, When not able to fully reflect the frequency and frequency variation in the partial frequency band due to nonlinear problems cause spurious frequency components would be unable to produce a better display fault vibration signal changes over time and wind speed. HHT method, EMD decomposition is adaptive, can effectively distinguish the frequency components and their changes.

\section{Helicopter rotor unbalance vibration signal analysis}

Unbalanced rotor helicopter failure experimental data were analyzed test bench. Experiments for three rotor blades, speed of $16 \mathrm{~Hz}$, conduct periodic sampling, the fault is set to pitch unbalanced.

Data indicates that the system never generates and failure to failure under certain wind speed (constant velocity) increases over time, the degree of fault, and the stronger the signal contains noise, non-steady signal. IMF component of the EMD obtained more significant. However, the low frequency signal component, the amplitude of the difference between the original signal level to $10^{-3}$, and its frequency components contain less than we want to analyze the frequency (16Hz). According to the characteristics IMF component, other components can be ignored. Hilbert transform when we take the first six natural frequencies and do it when the component spectrum and marginal spectrum. 

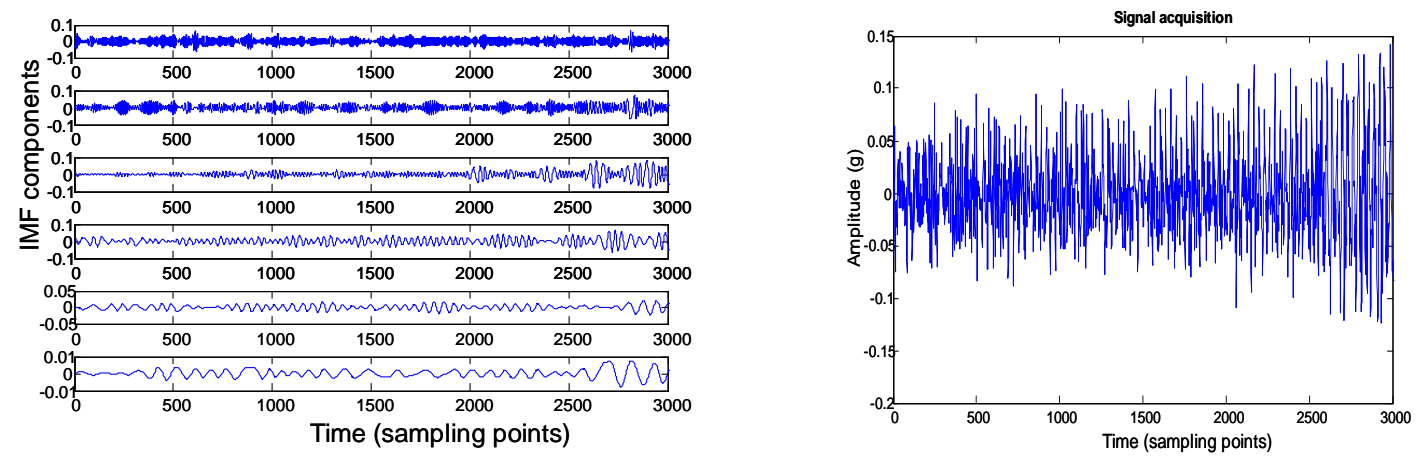

Fig. 3 sampled signal and intrinsic mode components

\section{Completeness and quadrature components of the test IMF.}

Completeness means that each component can be obtained by adding the decomposed nature of the original signal. IMF components from the decomposition formula (2) shows that, EMD its good completeness. In this experimental data, for the first six order to reconstruct the IMF, the reconstructed signal and the difference between the original data in the 10-3 level, you can determine which has the completeness of the original signal.

Orthogonality index ${ }^{[3]}: \quad I O=\sum_{t=0}^{T}\left(\sum_{i=1}^{n+1} \sum_{j=1}^{n+1} I M F_{i}(t) I M F_{j}(t) / X^{2}(t)\right)$

Among them:

$$
X(t)=\sum_{i=1}^{n+1} I M F_{i}(t), X^{2}(t)=\sum_{i=1}^{n+1} I M F^{2}(t)+2 \sum_{i=1}^{n+1} \sum_{j=1}^{n+1} I M F_{i}(t) I M F_{j}(t)
$$

Calculated from the first six orthogonal order index up to about $5 \%$, a minimum of $0.04 \%$, the correct data can be analyzed ${ }^{[4]}$.

\section{HHT processing and feature extraction.}

In the fault extraction process, marginal spectrum can effectively yield different fault different features at different frequencies or same, So marginal spectrum is characterized by rotor speed multiplier, Fig.4 shows the tab when unbalanced fault resulting Hilbert spectrum and marginal spectrum characteristics.

Hilbert marginal spectrum, when there is time away from the unbalanced rotor blade failure, hair experiment, frequency component of the body's vertical speed will produce 1,2,3 and 5 octave octave, and to a frequency-based, maximum vibration.

Fig.5 show the same speed, with anteversion, in the same direction (vertical), counterweight rotor unbalance and tab settings unbalanced fault resulting Hilbert. From the figure can be drawn, Hilbert spectrum was different fault on different octave showed different results, can be a good draw fault information.
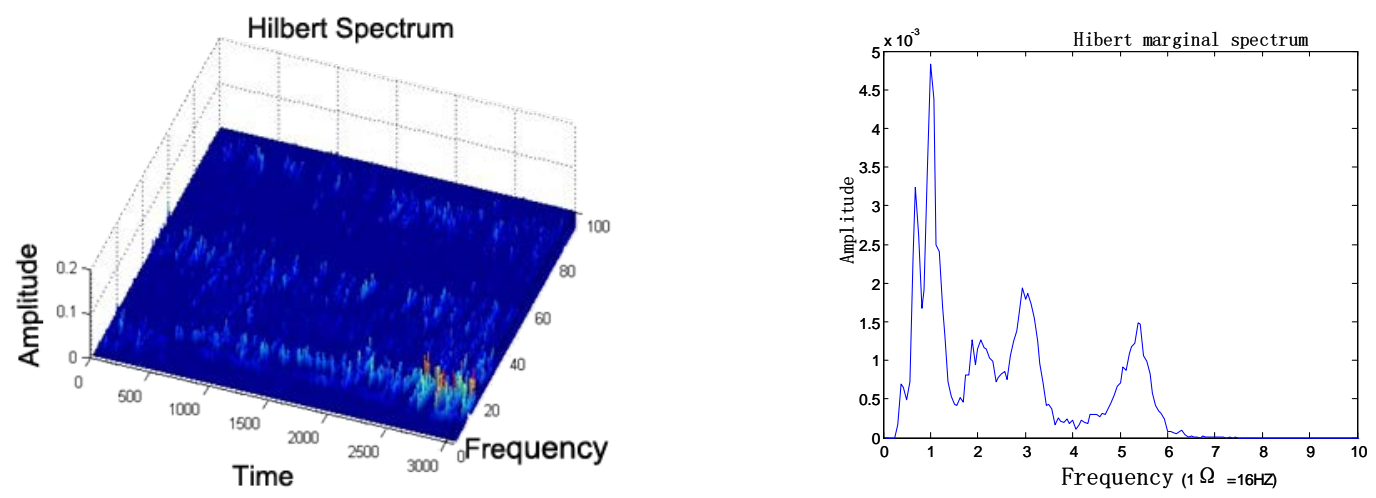

Fig.4 Experimental signal Hilbert spectrum and marginal spectrum 

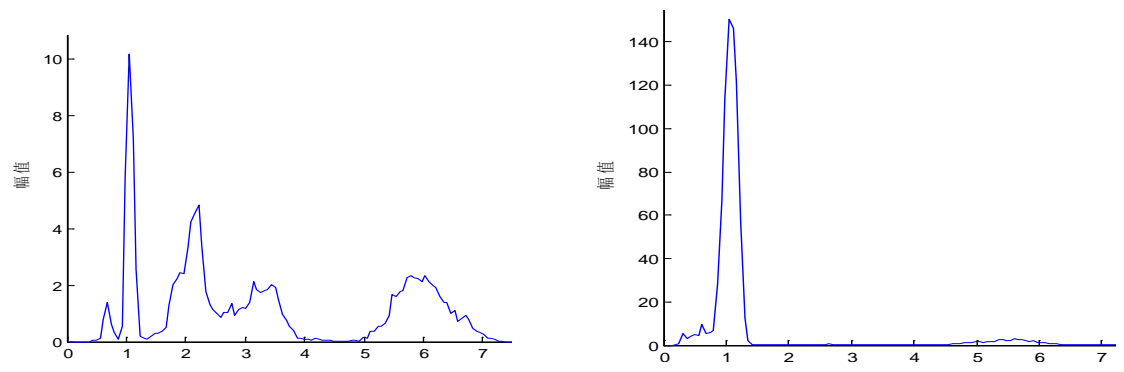

Fig. 5 counter weight (left) and pitch (right) unbalanced fault marginal

\section{Conclusion}

In this paper, the signal obtained from the simulation and analysis of the data obtained in the actual measurement: HHT method rotor unbalance fault feature extraction, To reflect changes in the vibration signal and distinct feature information can be extracted in different failure, Accurately reflect the change in the signal characteristics generated with the flight, in order to achieve fault monitoring and identification, diagnosis.

\section{References}

[1] Gaoyadong, Zhangzengchang: Body vibration signal with the theoretical basis of rotor unbalance fault diagnosis [J]. Vibration, Measurement \& Diagnosis (2003), P.23.

[2] Wang Zhuwen, Liu Jinghua, Yue Chongwang: The filtering Characteristics of HHT and its application acoustic log wave form signal processing, APPLIED GEOPHYSICS (2009), P.8-16.

[3] Yudejie, Chenjunsheng: Hilbert-Huang transform mechanical fault diagnosis [M]. Science Press.

[4] Norden E.Huang, Shen Z,Long S R er al: The empirical mode decomposition and the Hilbert spectrum for nonlinear and non-stationary time series analysis[J].Proc.R.Soc.Lond.A(1998),P. 903-995.

[5] Gabriel Rilling, Patrick Falandrin, Fellow: Brivate Empirical Mode Decomposition [J]. IEEE SINGNAL PROCESSING LETTERS (2007). 